Disponível em

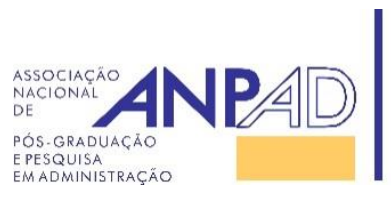
pp. 197-215, Mar./Abr. 2016

http://www.anpad.org.br/rac

RAC, Rio de Janeiro, v. 20, n. 2, art. 4,

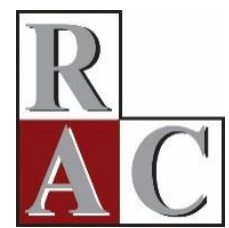

\title{
Impacto do Marketing Interno sobre a Orientação para o Mercado em Empresas Brasileiras
}

The Impact of Internal Marketing on the Market Orientation of Brazilian Companies 


\title{
Resumo
}

O conceito de marketing interno refere-se à visualização dos colaboradores como consumidores internos e assinala a necessidade de ouvir e satisfazer os colaboradores como requisito e satisfação dos colaboradores como requisitos para a satisfação dos clientes da empresa. Este artigo centra-se, assim, na influência do marketing interno na adoção de uma cultura empresarial orientada para os clientes, i.e., na orientação para o mercado. Com base na operacionalização do conceito de marketing interno de Lings e Greenley (2005), propõe-se um modelo conceitual das relações entre os conceitos de orientação para o mercado interno e orientação para o mercado. Os resultados de um estudo empírico baseado na aplicação de um questionário a 109 empresas brasileiras de diversos setores econômicos corroboram o modelo de investigação proposto. Desta forma, verificou-se que a adoção de um comportamento orientado para o mercado interno - nomeadamente as dimensões relativas à divulgação de informações e implementação de respostas - influencia a orientação para o mercado externo e suas dimensões (produção de informações, divulgação de informações e resposta).

Palavras-chave: marketing interno; orientação para o mercado interno; orientação para o mercado; gestão de pessoas; empresas brasileiras.

\begin{abstract}
The internal marketing concept refers to visualizing employees as internal consumers and emphasizes the need to listen to and satisfy employees as a requisite to satisfying company customers. This paper focuses, then, on the influence of internal marketing on the adoption of a corporate culture oriented to customers; i.e., a marketing orientation. Based on operationalization of the internal marketing concept from Lings and Greenley (2005), we propose a conceptual model for connections between the internal market orientation and market orientation concepts. The results of an empirical study based on a questionnaire applied to 109 Brazilian firms from several economic sectors corroborate the proposed model. The adoption of internal market oriented behavior, particularly the dimensions relative to the dissemination of information and implementation of responses, influences external market orientation and its dimensions (information production, information disclosure and response).
\end{abstract}

Key words: internal marketing; internal market orientation; market orientation; human resources management; Brazilian companies. 


\section{Introdução}

Diante da intensificação da concorrência e do ritmo acelerado das mudanças no ambiente de negócios, é fundamental que as empresas mantenham uma comunicação efetiva com os seus colaboradores e clientes, de forma a contribuir para a eficiência e qualidade de serviço da organização. $\mathrm{O}$ marketing interno assume grande importância neste contexto de crescente competitividade. Entendido como conceito, filosofia ou prática de gestão (Farias, 2010), o marketing interno refere-se à aplicação dos princípios do marketing ao contexto interno das organizações. A adoção de uma orientação para o marketing interno reflete, assim, em que medida as organizações se comprometem a produzir valor para os seus colaboradores, através de uma gestão eficaz das relações entre colaboradores, supervisores e gestores (Gounaris, 2006), para promover maior satisfação entre os funcionários, melhor serviço e, consequentemente, melhores resultados para o negócio.

Neste sentido, a abordagem de marketing interno contribui para o sucesso na organização no mercado externo. A orientação para o mercado consiste na adoção de uma cultura empresarial baseada na perspectiva do cliente (Deshpandé, Farley, \& Webster, 1993; Kohli \& Jaworski, 1990; Narver \& Slater, 1990; Ruekert, 1992; Shapiro, 1988), sendo um conceito basilar em marketing que tem merecido uma atenção crescente. Com base na contribuição seminal de Kohli e Jaworsky (1990), este conceito designa a orientação da empresa para a coleta, disseminação e utilização de informação sobre os clientes e o mercado. A literatura nesta área tem considerado que a orientação para o mercado produz valor para os consumidores e pode ser uma fonte sustentável de vantagem competitiva (Liao, Chang, Wu, \& Katrichis, 2011), tendo a relação positiva entre orientação para o mercado e o desempenho organizacional sido comprovada em numerosos estudos empíricos (Paim, Lara, \& Christino, 2011).

Assim, tanto o marketing interno como a orientação para o mercado assumem grande importância para o desempenho organizacional de uma organização. No entanto, a literatura sobre estes dois conceitos é ainda relativamente escassa. Relativamente à temática do marketing interno, de uma forma geral, as questões internas das organizações não têm sido estudadas na perspectiva do marketing (Tortosa, Moliner, \& Sánches, 2009). Farias (2010) sublinha a confusão existente na literatura causada pela indefinição acerca do conceito de marketing interno. Além disso, estudos empíricos sobre a prática em contextos nacionais específicos, incluindo sobre a experiência das empresas brasileiras, são também relativamente raros (Cassundé, Cassundé, Farias, \& Mendonça, 2014; Hemais, Oliveira, \& Casotti, 2013). Por exemplo, um estudo bibliométrico recentemente publicado identificou apenas 13 publicações brasileiras sobre este tema em bases de dados de referência no período 1961-2012 (Cassundé et al., 2014).

Também a literatura sobre orientação para o mercado, apesar da sua importância teórica e prática para o desempenho organizacional, permanece bastante limitada. Paim, Lara e Christino (2011) assinalam que os estudos empíricos têm sido realizados sobretudo em países industrializados.

Assim, este artigo visa analisar as práticas de marketing interno das empresas brasileiras, identificando o papel do marketing interno na promoção da orientação para o mercado externo. Este artigo está organizado da seguinte forma: na seção seguinte, a literatura relevante sobre marketing interno e orientação para o mercado é revista, culminando na apresentação de um modelo conceitual. $\mathrm{Na}$ seção três, é apresentada a metodologia do presente estudo, incluindo a operacionalização dos construtos. Os resultados do estudo empírico são analisados e discutidos na seção quatro. O artigo termina com a conclusão que enfatiza as principais contribuições deste estudo. 


\section{Revisão da Literatura}

Nesta seção, apresenta-se o enquadramento teórico relevante para este estudo, discutindo a literatura sobre os conceitos de marketing interno, orientação para o mercado e orientação para o mercado interno, a partir da qual se propõe o modelo conceitual de base deste estudo.

\section{Marketing interno}

O conceito de marketing interno surgiu na literatura somente na década de 1970 (Berry, Hensel, \& Burke, 1976). Berry, Hensel e Burke (1976) referem que a satisfação dos colaboradores é tão importante quanto a satisfação dos clientes, constituindo um requisito para a qualidade dos serviços. A capacidade de satisfação dos clientes por parte da empresa depende, assim, do nível de satisfação e do grau de orientação para os clientes e o mercado dos funcionários. Marketing interno é, então, definido como a visualização dos colaboradores como consumidores internos (Berry et al., 1976). O enfoque na satisfação dos trabalhadores nesta nova abordagem da gestão dos colaboradores pode ser atribuído ao fato de que, nos serviços, muito do que os clientes compram corresponde a trabalho, ou ações e desempenho humano (Rafiq \& Ahmed, 2000). Desta forma, em muitos casos, para os clientes, o serviço é o funcionário. A ferramenta fundamental para manter a satisfação dos colaboradores segundo esta abordagem é tratá-los como clientes internos, que trocam serviços entre si.

No entanto, apesar da filosofia dos colaboradores como clientes ser bastante atrativa, diversos autores apontaram potenciais problemas nesta conceitualização (Piercy, 1995; Rafiq \& Ahmed, 1993).

Outra abordagem foi proposta por Grönroos (1994) que sublinha que, para além do impacto na compra e na decisão de repetição da compra, a interação do vendedor com o cliente providencia uma oportunidade de marketing para a organização, fazendo dos colaboradores não pertencentes à área do marketing, funcionários de marketing em part-time (George, 1990; Grönroos, 1994). Assim, é necessário que os trabalhadores tenham orientação para o cliente e espírito comercial, sendo que, para este autor, o objetivo do marketing interno é fazer com que os funcionários estejam motivados e focalizados no cliente. Neste sentido, o marketing interno é um pré-requisito para a comercialização externa com sucesso e algumas atividades de marketing externo tradicional podem ser utilizadas conjuntamente com a formação e outras atividades de desenvolvimento pessoal tradicionais (Grönroos, 1995).

Uma outra conceitualização de marketing interno o reconhece como um veículo da implementação da estratégia das organizações (Rafiq \& Ahmed, 2000), baseando-se no potencial do marketing interno enquanto mecanismo de integração interfuncional dentro da organização.

Assim, apesar de não existir consenso sobre o significado conceitual e operacional do marketing interno (Farias, 2010), a abordagem iniciada por Berry et al. (1976) implica ações como atração e seleção de funcionários, a socialização destes, capacitação, participação na tomada de decisões e estabelecimento de informações precisas, bem como abertura entre funcionários e administradores. No entanto, essas ações ocorrem num quadro específico orientado para o cliente final, o que explica por que o marketing interno é diferente da gestão de recursos humanos (Gounaris, 2008).

\section{Orientação para o mercado}

O conceito de orientação para o mercado tem origem no conceito de marketing (Raaij \& Stoelhorst, 2008). Esta filosofia tem sido uma pedra angular da disciplina de marketing. O conceito tornou-se bastante apelativo para os gestores e tem sido uma das ideias mais influentes do marketing (Raaij \& Stoelhorst, 2008). Um número crescente de estudos tem analisado a sua ligação com diferentes conceitos como desempenho, inovação, aprendizagem, qualidade e competitividade (Liao et al., 2011). As atividades que envolvem uma efetiva orientação para o mercado são consideradas requisitos para as 
organizações serem capazes de fornecer produtos e serviços superiores em resposta às necessidades dos clientes (Atuahene-Gima, 1996; Liao et al., 2011).

As primeiras definições de orientação para o mercado devem-se a Shapiro (1988), Kohli e Jaworski (1990), Narver e Slater (1990), Ruekert (1992) e Deshpandé, Farley e Webster (1993).

Para Shapiro (1988), uma empresa terá uma orientação para o mercado se as informações sobre todas as influências de compra importantes atravessarem todas as funções corporativas, se as decisões estratégicas e táticas forem decididas com a participação de todas as funções e divisões e se estas coordenarem as decisões e as executarem com um sentido de compromisso. Para Kohli e Jaworski (1990), a orientação para o mercado consiste na geração de informação de mercado sobre as necessidades atuais e futuras dos clientes, na disseminação dessa informação pela organização e na capacidade de implementação de respostas adequadas. Por seu lado, segundo Narver e Slater (1990), a orientação para o mercado é "a cultura empresarial, que de forma mais eficaz e eficiente, cria os comportamentos necessários para a criação de valor superior para os clientes" (p. 21) e inclui três componentes comportamentais: a orientação para o cliente, a orientação para os concorrentes e a coordenação interfuncional.

Ruekert (1992) descreve o nível de orientação para o mercado como o grau em que a unidade de negócios obtém e utiliza informações de clientes para desenvolver e implementar uma estratégia ágil para responder às necessidades e desejos dos clientes. Deshpandé et al. (1993) relatam que a orientação para o cliente é o conjunto de crenças que coloca o interesse do cliente em primeiro lugar, a fim de desenvolver uma empresa rentável a longo prazo.

Estas diferentes perspectivas enfatizam a importância da informação de mercado. A orientação para o mercado consiste na coleta, disseminação e resposta integrada a esta informação por parte das organizações.

\section{Orientação para o mercado interno}

Os recentes avanços na área de marketing interno levaram à identificação de comportamentos de gestão específicos associados a essa filosofia (Lings \& Greenley, 2010), e à conceitualização da orientação para o mercado interno (Gounaris, 2006; Lings, 2004; Lings \& Greenley, 2005).

A orientação para o mercado interno estimula as organizações a desenvolver um relacionamento eficaz com os seus funcionários, tendo como base o compromisso de lhes oferecer um valor superior, compreendendo e satisfazendo as suas necessidades manifestas e latentes, de forma a criar valor para os clientes da empresa (Gounaris, 2006).

A definição de orientação para o mercado interno de Lings e Greenley (2005) inclui dimensões referentes à geração de informações sobre o mercado interno, à divulgação de informações e à implementação de respostas a essa informação. A geração de informação decorre das interações quotidianas e da proximidade física entre colaboradores e os gestores, oferecendo, assim, oportunidades adicionais para a geração de informação, com base nas atitudes, valores e situações individuais.

Relativamente à informação formal, esta tem uma manifestação bidimensional através da utilização de meios de comunicação escritos, como os questionários e estudos de satisfação no trabalho, e interações face-to-face, como entrevistas, avaliações e reuniões, sendo estes dois métodos complementares (Lings \& Greenley, 2005). Segundo estes autores, a divulgação da informação é um fator fundamental para alinhar as atitudes e comportamentos dos colaboradores com os objetivos da organização. A proximidade entre gestores e colaboradores aumenta as oportunidades para a comunicação, proporcionando a oportunidade para a recolha de informações sobre os anseios e necessidades dos empregados e também para divulgar informações.

Relativamente à implementação de respostas, Lings e Greenly (2005) enfatizam que a concepção e implementação destas devem responder às necessidades e desejos dos colaboradores. Esta dimensão 
envolve atividades como o desenvolvimento de sistemas de recompensas individualizados, avaliação de desempenho, mudanças de horário de trabalho e oportunidades de formação.

\section{Modelo conceitual e hipóteses}

Da revisão da literatura realizada, decorre a proposição de que a orientação para o mercado interno influencia a orientação para o mercado (Gounaris, Vassilikopoulou, \& Chatzipanagiotou, 2010; Lings \& Greenley, 2005, 2010; Zaman, Javaid, Arshad, \& Bibi, 2012), pelo que se propõe o seguinte modelo de investigação (Figura 1).

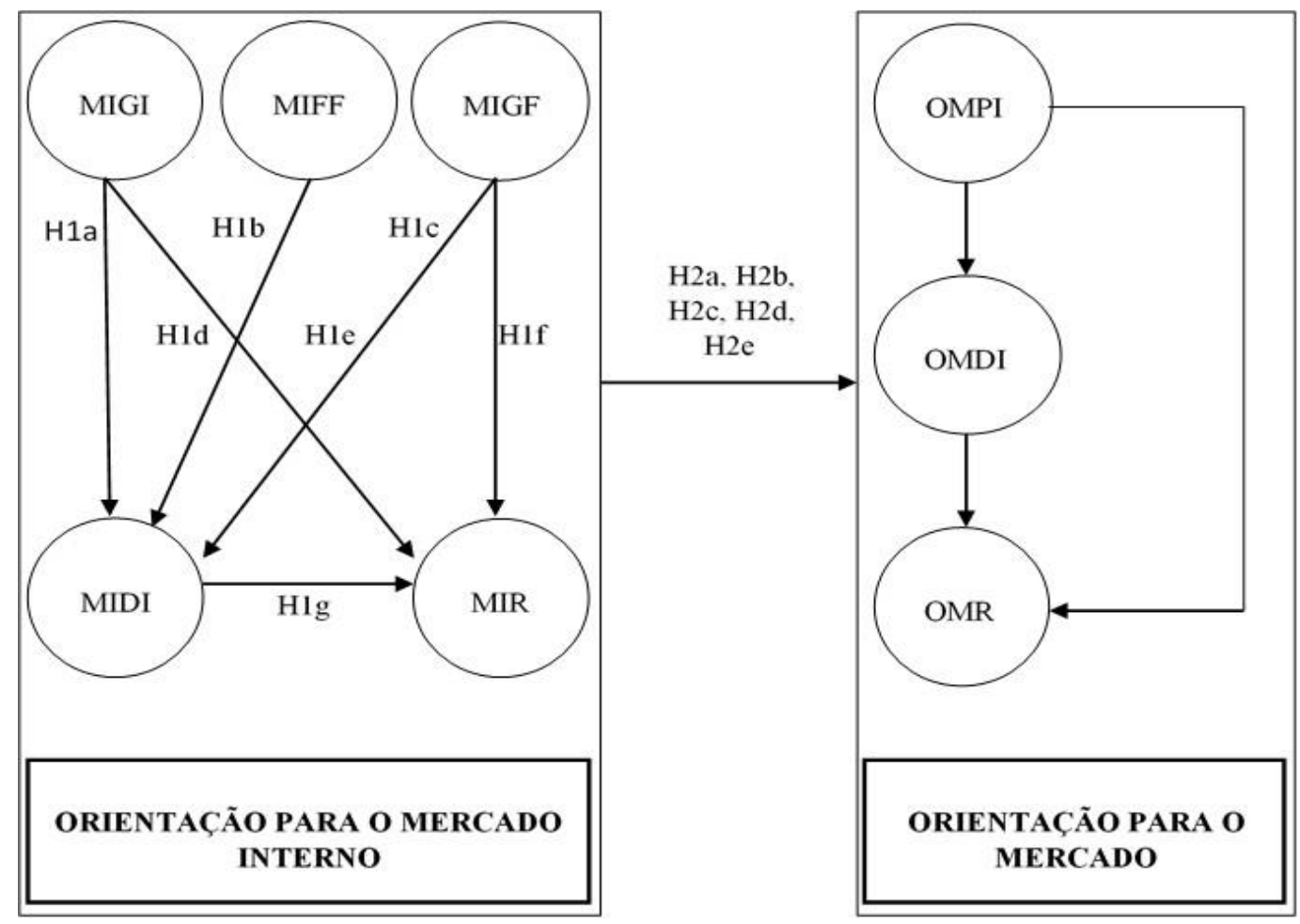

Figura 1. Modelo de Investigação

MIGI - Orientação para o mercado interno: Geração de informação informal; MIFF - Orientação para o mercado interno: Geração de informação formal face-to-face; MIGF - Orientação para o mercado interno: Geração de informação formal escrita; MIDI - Orientação para o mercado interno: Divulgação de informação; MIR - Orientação para o mercado interno: Implementação de respostas; OMPI - Orientação para o mercado: Produção de informação; OMDI - Orientação para o mercado: Divulgação de informação; OMR - Orientação para o mercado: Resposta. Fonte: adaptado de Lings, I. N., \& Greenley, G. E. (2010). Internal market orientation and market-oriented behaviours. Journal of Service Management, 21(3), 321-343. doi: 10.1108/09564231011050788

A geração de informação acerca do mercado interno precede a divulgação dessas mesmas informações, pelo que a geração e disseminação de informação tendem a preceder a criação de respostas de valor para o mercado interno (Lings \& Greenley, 2010). Seguindo a linha de orientação do mercado de Jaworski e Kohli (1993), estas dimensões relativas às geração e difusão da informação estão relacionadas. São propostas as seguintes hipóteses quanto ao conceito de orientação para o mercado interno:

H1a. As capacidades de geração de informação informal do mercado interno (MIGI) têm um impacto positivo nas capacidades de divulgação interna (MIDI).

H1b. As capacidades de geração de informação formal face-to-face do mercado interno (MIFF) aumentam as capacidades de divulgação interna (MIDI).

H1c. As capacidades de geração de informação formal escrita do mercado interno (MIGF) aumentam as capacidades de divulgação interna (MIDI). 
H1d. As capacidades de geração de informação informal do mercado Interno (MIGI) aumentam as capacidades de implementação de respostas (MIR).

H1e. As capacidades de geração de informação formal face-to-face do mercado interno (MIFF) aumentam as capacidades de implementação de respostas (MIR).

H1f. As capacidades de geração de informação formal escrita do mercado interno (MIGF) aumentam as capacidades de implementação de respostas (MIR).

H1g. As capacidades de divulgação interna da informação (MIDI) aumentam as capacidades de implementação de respostas (MIR).

Orientação para o mercado interno e orientação para o mercado são dois conceitos interrelacionados (Gounaris et al., 2010; Lings \& Greenley, 2009, 2010). A necessidade de as empresas adotarem uma orientação para o mercado interno com vistas à implementação com sucesso da orientação para o mercado tem sido amplamente discutida na literatura (Ahmed \& Rafiq, 2011). A geração de informação, a comunicação e capacidade de resposta dos funcionários proporcionadas pela orientação para o mercado interno catalisam a transferência de informações entre os funcionários da linha de frente e os gestores. Como resultado, a orientação para o mercado interno deverá ter um impacto positivo sobre a dimensão de geração de informações de orientação para o mercado e sobre os resultados da organização (Rafiq \& Ahmed, 1993).

Consequentemente, espera-se que a orientação para o mercado interno tenha um impacto positivo sobre a dimensão da capacidade de resposta da orientação para o mercado (Caruana \& Calleya, 1998; Gounaris et al., 2010; Lings \& Greenley, 2009, 2010; Naude, Desai, \& Murphy, 2003; Piercy, 1995; Zaman et al., 2012). Assim, propõem-se as seguintes hipóteses quanto ao inter-relacionamento entre os conceitos de orientação para o mercado interno e orientação para o mercado:

H2a. As capacidades de geração de informação informal do mercado interno (MIGI) aumentam as capacidades de orientação para o mercado (OMPI, OMDI e OMR).

H2b. As capacidades de geração de informação formal face-to-face do mercado interno (MIFF) aumentam as capacidades de orientação para o mercado (OMPI, OMDI e OMR).

H2c. As capacidades de geração de informação formal escrita do mercado interno (MIGF) aumentam as capacidades de orientação para o mercado (OMPI, OMDI e OMR).

H2d. As capacidades de divulgação interna da informação (MIDI) aumentam as capacidades de orientação para o mercado (OMPI, OMDI e OMR).

H2e. As capacidades de implementação de respostas (MIR) aumentam as capacidades de orientação para o mercado (OMPI, OMDI e OMR).

A maioria dos estudos sobre os efeitos da orientação para o mercado a tem operacionalizado como um construto unidimensional composto por três componentes. Contudo, os autores da escala, Jaworski e Kohli (1993), sugerem que é necessário examinar as relações causais entre a geração de informações, a disseminação e capacidade de resposta. Assim, propõem-se as seguintes hipóteses quanto ao conceito de orientação para o mercado:

H3a. As capacidades de produção de informação (OMPI) aumentam as capacidades de divulgação da informação (OMDI).

H3b. As capacidades de divulgação da informação (OMDI) aumentam as capacidades de resposta (OMR).

H3c. As capacidades de produção de informação (OMPI) aumentam as capacidades de resposta (OMR). 


\section{Metodologia}

Este estudo seguiu uma abordagem quantitativa, com obtenção dos dados através da aplicação de um questionário. O questionário foi enviado para uma amostra não aleatória de 900 empresas brasileiras obtida através de uma diretoria de empresas. O questionário foi endereçado ao proprietário da empresa ou a algum dos administradores ou gestores de primeira linha. A coleta dos dados foi realizada durante o mês de agosto de 2013, tendo sido enviado um e-mail para as empresas, explicando quais os objetivos do trabalho, contendo um link para o questionário eletrônico elaborado na plataforma Qualtrics.

Foram recebidos 109 questionários, correspondendo a uma taxa de resposta de 12,1\%. Consideramos esta taxa de resposta bastante satisfatória, dada a dificuldade em conseguir a participação de gestores de topo. De fato, as taxas de resposta em contextos industriais são muito baixas. Por exemplo, Harzing (2000) reporta que a taxa de resposta a questionários que não sejam precedidos ou seguidos por um telefonema varia entre 6 e $16 \%$.

\section{Operacionalização dos construtos}

Para operacionalizar os construtos, foram identificadas escalas de medida já validadas em estudos anteriores.

Em relação à orientação para o mercado, foram propostas várias escalas para medir o construto. Como nos focamos na perspectiva comportamental da orientação para o mercado, a escala utilizada neste estudo é a MARKOR (Kohli, Jaworski, \& Kumar, 1993). Esta escala identifica o grau em que uma organização realiza atividades de produção de informação no que tange a todos os seus departamentos, a maneira como esta informação é disseminada vertical e horizontalmente e a forma como desenvolve e implementa os programas de marketing interno e externo com base na informação gerada, ou seja, a resposta ao mercado. A escala MARKOR é constituída por vinte variáveis (Tabela 1), alocadas em três dimensões: produção de informação de mercado (OMPI); divulgação da informação de mercado (OMDI); e resposta ao mercado (OMR). Salienta-se ainda que uma versão em português desta escala já foi validada no Brasil por Sampaio (2000).

Tabela 1

\section{Dimensões da Escala MARKOR e Respetivos Itens}

\section{Produção de informação (OMPI)}

1. Nesta empresa, pelo menos uma vez ao ano identificamos que produtos ou serviços nossos clientes necessitarão no futuro.

2. Nesta empresa, nós próprios fazemos muita pesquisa de mercado.

3. Somos lentos para detectar mudanças nas preferências de nossos clientes nos produtos*.

4. Nós pesquisamos os clientes pelo menos uma vez ao ano para avaliar a qualidade de nossos serviços.

5. Somos lentos ao detectar mudanças fundamentais em nosso ramo de atividade (por exemplo: concorrência, tecnologia, legislação)*. 


\section{Tabela 1 (continuação)}

\section{Divulgação de informação (OMDI)}

6. Nós revisamos periodicamente os prováveis efeitos das mudanças em nosso ambiente empresarial (por exemplo, legislação, mudanças na economia) sobre nossos clientes.

7. Nós temos reuniões interdepartamentais pelo menos uma vez por trimestre para discutir tendências e desenvolvimento do mercado.

8. O pessoal de marketing em nossa empresa investe tempo discutindo com outros departamentos sobre as necessidades futuras dos clientes.

9. Quando algo relevante acontece a um cliente importante, toda a empresa sabe sobre o ocorrido em curto intervalo de tempo.

10. Dados sobre a satisfação do cliente são disseminados regularmente em todos os níveis hierárquicos nesta empresa.

11. Quando um departamento descobre algo importante sobre os concorrentes, ele é lento para alertar os outros departamentos*.

\section{Resposta (OMR)}

12. Nós demoramos muito tempo para decidir como responder às mudanças de preços dos nossos concorrentes*.

13. Tendemos a ignorar mudanças nas necessidades de produtos ou serviços dos nossos clientes*.

14. Nós revisamos periodicamente nossos esforços de desenvolvimento de novos produtos ou serviços para assegurar que eles estão de acordo com o que os clientes desejam.

15. Diversos departamentos ou áreas de nossa empresa encontram-se periodicamente para planejar uma resposta às mudanças que ocorrem em nosso ambiente de negócios.

16. Se um importante concorrente lançasse uma campanha intensiva dirigida a nossos clientes, nós implementaríamos uma resposta imediatamente.

17. As atividades dos diferentes departamentos nesta empresa são bem coordenadas.

18. As reclamações dos clientes "não têm ouvidos" nesta empresa*.

19. Mesmo se nós formulássemos um ótimo plano de marketing, nós provavelmente não seríamos capazes de implementá-lo em tempo adequado*.

20. Quando sabemos que os clientes gostariam que modificássemos um produto ou serviço, os departamentos envolvidos fazem esforços combinados para efetuar a modificação.

Nota. * Itens reversos.

Relativamente ao construto orientação para o mercado interno, será usada a escala desenvolvida por Lings e Greenley (2005). Esta escala inclui dezesseis itens referentes a cinco dimensões da orientação para o mercado interno: três dimensões referentes à geração de informações sobre o mercado interno (Geração de informação informal - MIGI, Geração de informação formal face-to-face - MIFF, e Geração de informação formal escrita - MIGF), divulgação de informações (MIDI) e implementação de respostas (MIR). A Tabela 2 lista os itens que constituem a escala. 
Tabela 2

\section{Dimensões da Escala de Orientação para o Mercado Interno e Respetivos Itens}

\section{Geração de informação informal (MIGI)}

1. Quando estou no trabalho, procuro identificar as expectativas dos colaboradores, o que esperam da empresa.

2. Quando estou no trabalho, ao notar um dos colaboradores agindo de forma diferente do normal, procuro descobrir se existe um problema.

3. Quando estou no trabalho, procuro identificar o grau de satisfação deles sobre o trabalho.

\section{Geração de informação formal face-to-face (MIFF)}

4. Quando estou no trabalho, procuro acompanhar a equipe para identificar a performance do trabalho.

5. Nesta empresa, temos avaliações de pessoal regulares em que discutimos o que os funcionários querem.

6. Nesta empresa, a gerência se reúne com os colaboradores pelo menos uma vez por ano para descobrir as suas expectativas de trabalho para o futuro.

\section{Geração de informação formal escrita (MIGF)}

7. Nesta empresa, a gerência relaciona-se diretamente (por meio de conversas e pesquisas) com os colaboradores para descobrir como torná-los mais satisfeitos.

8. Nesta empresa fazemos muita pesquisa sobre o mercado interno.

9. Nesta empresa, aplicamos um questionário sobre os colaboradores, pelo menos uma vez por ano para avaliar a qualidade do trabalho.

\section{Divulgação de informações (MIDI)}

10. Nesta empresa, aplicamos um questionário sobre os colaboradores para identificar influências sobre o comportamento pelos sindicatos, clientes e mercado externo.

11. Nesta empresa, eu regularmente reúno a equipe para falar sobre as questões relacionadas à organização.

12. Nesta empresa, eu regularmente reporto aos colaboradores as questões que afetam o ambiente de trabalho.

13. Nesta empresa, temos reuniões regulares com os colaboradores de todos os níveis.

\section{Implementação de respostas (MIR)}

14. Nesta empresa, quando descobrimos que os funcionários estão descontentes com a nossa supervisão ou gerência, tomamos ações corretivas.

15. Nesta empresa, quando se percebe que os trabalhadores gostariam que modificassem as suas condições de trabalho, os departamentos se esforçam para buscar uma adequação nesse sentido.

16. Nesta empresa, fazemos as mudanças devidas quando o feedback dos colaboradores indica que eles estão insatisfeitos com o status quo.

\section{Análise e Discussão dos Resultados}

Nesta seção, apresentamos os resultados obtidos, começando com a caracterização da amostra. Em seguida, apresentamos as caraterísticas psicométricas dos construtos de forma a proceder à análise das hipóteses desenvolvidas.

\section{Caracterização da amostra}

A amostra é constituída maioritariamente por empresas pertencentes ao setor dos serviços $(62,2 \%)$. Em termos do número de trabalhadores, 55,1\% das empresas têm 250 ou mais trabalhadores, 
$15,3 \%$ têm entre 50 e 249 trabalhadores e 16,6\% possuem entre 10 e 49 colaboradores. Quanto à formação, 34,8\% das empresas têm pelo menos 50\% de trabalhadores com formação superior, 27,4\% têm entre $1 \%$ e $9 \%$ de trabalhadores com formação superior e $21,1 \%$ das empresas têm entre $10 \%$ e $24 \%$ de colaboradores com educação superior.

O tempo médio de atividade das empresas incluídas no estudo é 13,7 $\pm 15,8$ anos, variando entre 1 mês e 70 anos. Em termos de estrutura empresarial, 58,8\% das empresas pertencem a um grupo empresarial, dos quais $91,2 \%$ são grupos brasileiros e os demais grupos têm origem em países como os Estados Unidos, Alemanha, Grã-Bretanha e Chile. Os principais mercados dos bens ou serviços vendidos pelas empresas da amostra são os mercados nacional $(56,7 \%)$ e local/regional $(37,1 \%)$. No que respeita ao volume de negócios no último ano, constata-se que $37,1 \%$ das empresas faturaram mais de 50 milhões de reais, $19,1 \%$ faturaram entre 10 e 50 milhões de reais, 20,2\% tiveram um volume de negócios entre 2 e 9 milhões de reais e em $23,6 \%$ das empresas este foi inferior a 2 milhões de reais (Tabela 3).

Tabela 3

\section{Caracterização da Amostra}

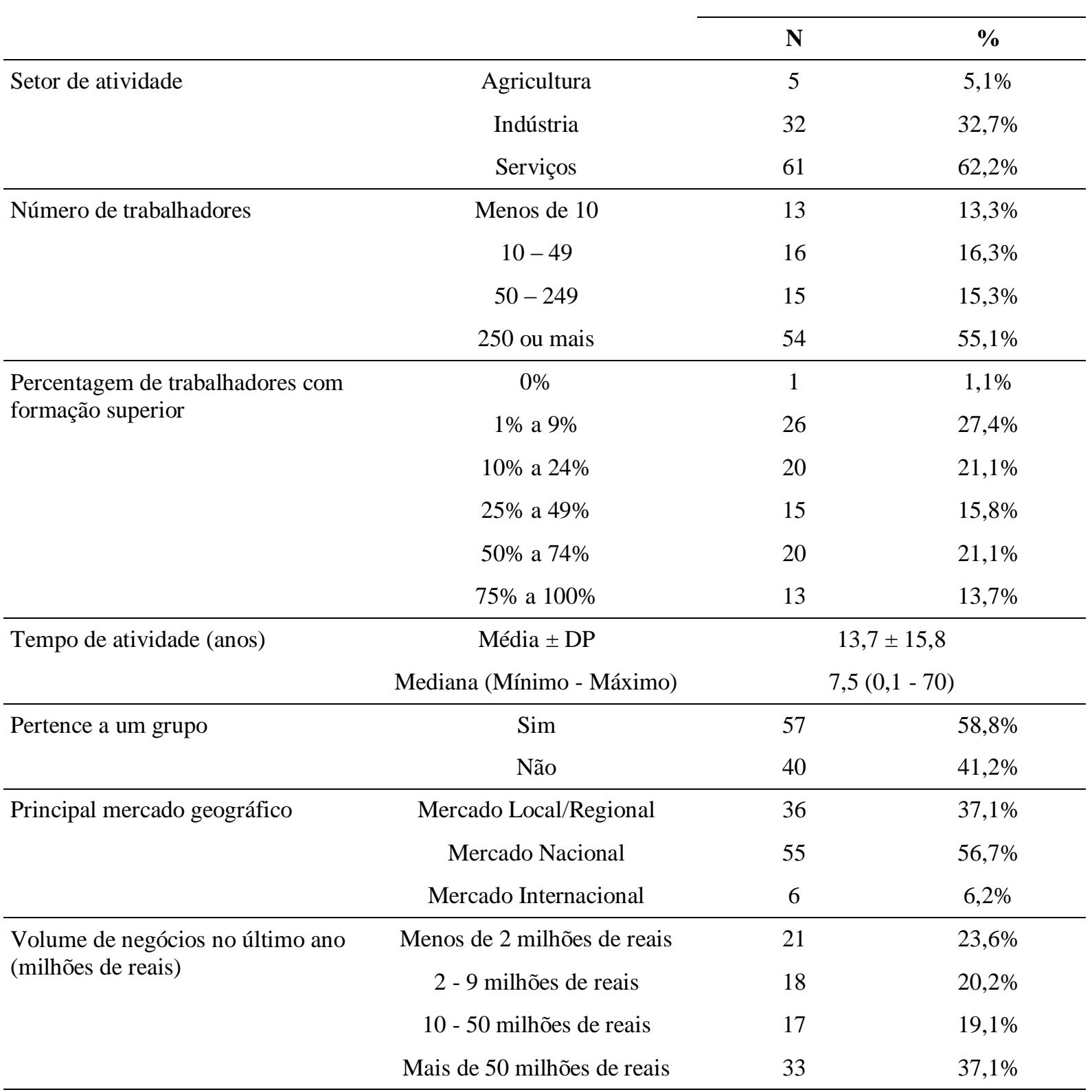

Nota. Fonte: dados da pesquisa. 


\section{Características psicométricas dos construtos}

Analisando as propriedades psicométricas dos construtos em estudo e respectivas dimensões (Tabela 4), constata-se que os Alphas de Cronbach variam entre 0,746 e 0,904 nas dimensões da Orientação para o mercado interno e entre 0,599 e 0,676 nas dimensões alusivas à Orientação para o mercado, constatando-se a existência de confiabilidade destes construtos.

Diante destes resultados, infere-se, para as diversas dimensões de ambas as escalas em estudo, a existência de caraterísticas psicométricas aceitáveis para a sua utilização. Estes resultados corroboram as caraterísticas psicométricas das duas escalas reveladas em outros estudos, nomeadamente no estudo de Lings e Greenley (2010).

Tabela 4

Coeficiente de Correlação entre os Construtos (Alphas de Cronbach na Diagonal)

\begin{tabular}{ccccccccc} 
& MIGI & MIFF & MIGF & MIDI & MIR & OMPI & OMDI & OMR \\
\hline MIGI & 0,904 & & & & & & & \\
MIFF & $0,635^{* *}$ & 0,746 & & & & & & \\
MIGF & $0,421^{* *}$ & $0,729 * *$ & 0,804 & & & & & \\
MIDI & $0,590^{* *}$ & $0,804^{* *}$ & $0,793^{* *}$ & 0,824 & & & & \\
MIR & $0,401^{* *}$ & $0,718^{* *}$ & $0,716^{* *}$ & $0,734^{* *}$ & 0,879 & & & \\
OMPI & $0,196^{*}$ & $0,237^{*}$ & $0,217^{*}$ & 0,160 & 0,140 & 0,676 & & \\
OMDI & 0,169 & $0,304^{* *}$ & $0,413^{* *}$ & $0,390^{* *}$ & $0,336^{* *}$ & $0,450^{* *}$ & 0,599 & \\
OMR & $0,326^{* *}$ & $0,360^{* *}$ & $0,359^{* *}$ & $0,418^{* *}$ & $0,433^{* *}$ & $0,369^{* *}$ & $0,549 * *$ & 0,614 \\
\hline
\end{tabular}

Nota. Fonte: dados da pesquisa.

$* p<.05 ; * * p<.01$.

\section{Análise das hipóteses}

Para a validação das hipóteses, foram estimados modelos de regressão linear múltipla, estimados pelo método dos mínimos quadrados. A Tabela 5 apresenta os resultados relativos às hipóteses em que intervêm as dimensões da Orientação para o mercado interno. Foram estimados dois modelos de regressão (Modelo 1 e Modelo 2), tendo o primeiro o objetivo de avaliar as hipóteses H1a, H1b e H1c, e o segundo, avaliar as hipóteses H1d, H1e, H1f e H1g.

Tabela 5

Regressões Lineares

\begin{tabular}{ccc} 
& ${\text { Modelo } 1^{\mathrm{a}}}^{\mathrm{a}}$ & ${\text { Modelo } 2^{\mathrm{b}}}$ \\
\hline Constante & $-0,11(0,24)$ & $0,49(0,33)$ \\
MIGI & $0,19(0,07)^{* *}$ & $-0,15(0,10)$ \\
MIFF & $0,37(0,08)^{* *}$ & $0,39(0,13)^{* *}$ \\
MIGF & $0,42(0,06)^{* *}$ & $0,27(0,11)^{*}$ \\
MIDI & & $0,35(0,13)^{*}$ \\
\hline
\end{tabular}


Tabela 5 (continuação)

\begin{tabular}{ccc}
\cline { 2 - 3 } & Modelo $1^{\mathrm{a}}$ & ${\text { Modelo } 2^{\mathrm{b}}}^{\mathrm{a}}$ \\
\hline $\mathrm{R}^{2}$ & 0,754 & 0,622 \\
$\mathrm{R}^{2}$ ajustado & 0,747 & 0,608 \\
$\mathrm{~F}$ & $109,50^{* *}$ & $43,61^{* *}$ \\
\hline
\end{tabular}

Nota. Fonte: dados da pesquisa.

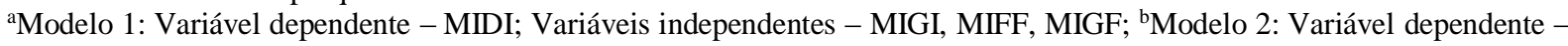
MIR; Variáveis independentes - MIGI, MIFF, MIGF, MIDI.

$* p<.05 ; * * p<.01$.

Estes resultados permitiram suportar as hipóteses: H1a; H1b; H1c; H1e, H1f e H1g. São assim suportadas as hipóteses segundo as quais as capacidades de geração de informação informal (MIGI), formal face-to-face (MIFF) e escrita (MIGF) sobre o mercado interno têm um impacto positivo estatisticamente significativo sobre as capacidades de divulgação interna da informação (MIDI). Para além disso, as capacidades de geração de informação formal face-to-face (MIFF), informação formal escrita (MIGF) e divulgação de informação (MIDI) sobre o mercado interno aumentam significativamente as capacidades de implementação de respostas (MIR). A Hipótese H1d, que propunha que as capacidades de geração de informação informal afetavam as capacidades de implementação de respostas, não foi suportada. Estes resultados corroboram parcialmente os resultados de Lings e Greenley (2010) e de Jaworski e Kohli (1993), constatando-se que a geração no mercado interno de informação afeta a divulgação dessas mesmas informações, sendo também possível atestar que a disseminação de informação afeta a implementação de respostas de valor para o mercado interno.

Relativamente ao impacto das dimensões da Orientação para o mercado interno sobre as dimensões da Orientação para o mercado (H2a, H2b, H2c, H2d e H2e), e às relações causais das dimensões da Orientação para o mercado (H3a, H3b e H3c), foram estimados três modelos de regressão (Modelo 3, Modelo 4 e Modelo 5) (Tabela 6).

Tabela 6

Regressões Lineares

\begin{tabular}{cccc} 
& Modelo 3 $^{\mathrm{a}}$ & Modelo $^{\mathrm{b}}$ & Modelo $^{\mathrm{c}}$ \\
\hline Constante & $2,42(0,38)$ & $1,93(0,38)$ & $1,48(0,31)$ \\
MIGI (H2a) & $-0,05(0,12)$ & $0,12(0,10)$ & $0,13(0,08)$ \\
MIFF (H2b) & $-0,08(0,15)$ & $0,21(0,13)$ & $-0,08(0,09)$ \\
MIGF (H2c) & $0,22(0,12)$ & $0,07(0,11)$ & $-0,08(0,08)$ \\
MIDI (H2d) & $0,20(0,16)$ & $-0,25(0,14)$ & $0,07(0,10)^{*}$ \\
MIR (H2e) & $0,04(0,11)^{* *}$ & $0,07(0,10)^{* *}$ & $0,17(0,07)^{* *}$ \\
OMDI & & $0,41(0,08)^{* *}$ & $0,29(0,07)^{* *}$ \\
OMPI & & & $0,13(0,07)^{* *}$ \\
\hline $\mathrm{R}^{2}$ & 0,187 & 0,255 & 0,419 \\
$\mathrm{R}^{2}$ ajustado & 0,148 & 0,212 & 0,379 \\
$\mathrm{~F}$ & $4,82^{* *}$ & $5,93^{* *}$ & $10,60^{* *}$ \\
\hline
\end{tabular}

Nota. Fonte: dados da pesquisa.

${ }^{a}$ Modelo 3: Variável dependente - OMDI; Variáveis independentes - MIGI, MIFF, MIGF, MIDI; ${ }^{b}$ Modelo 4: Variável dependente - OMPI; Variáveis independentes - MIGI, MIFF, MIGF, MIDI, OMDI; ${ }^{c}$ Modelo 5: Variável dependente - OMR; Variáveis independentes - MIGI, MIFF, MIGF, MIDI, OMDI, OMPI.

$* p<.05 ; * * p<.01$.

RAC, Rio de Janeiro, v. 20, n. 2, art. 4, pp. 197-215, Mar./Abr. 2016

www.anpad.org.br/rac (oc) 
Os resultados quanto ao impacto da orientação para o mercado interno sobre a orientação para o mercado são, assim, mistos. A hipótese H2e foi suportada, dado que as capacidades de implementação de respostas ao mercado interno (MIR) têm um impacto em todas as dimensões da orientação para o mercado, aumentando estatisticamente as capacidades de produção de informação (OMPI), as capacidades de divulgação da informação (OMDI) e as capacidades de resposta (OMR). Por um lado, a hipótese $\mathrm{H} 2 \mathrm{~d}$ foi parcialmente validada: as capacidades de divulgação interna da informação (MIDI) do mercado interno aumentam estatisticamente uma das vertentes da orientação para o mercado externo, especificamente as capacidades de resposta no mercado (OMR). No entanto, não se comprovou que as capacidades de geração de qualquer tipo de informação sobre o mercado interno tenham um impacto na orientação para o mercado externo (H2a, H2b e H2c).

Estes resultados são parcialmente concordantes com os relatados nos estudos de Caruana e Calleya (1998), Gounaris, Vassilikopoulou e Chatzipanagiotou (2010), Lings e Greenley (2009, 2010), Naude, Desai e Murphy (2003), Piercy (1995) e Zaman, Javaid, Arshad e Bibi (2012), que verificaram que a orientação para o mercado interno tem um impacto positivo sobre a capacidade de resposta da orientação para o mercado.

Finalmente, quanto às relações causais entre as dimensões da orientação para o mercado, observou-se que (a) as capacidades de produção de informação (OMPI) aumentam significativamente as capacidades de divulgação da informação (OMDI) e as capacidades de resposta (OMR), e (b) as capacidades de divulgação da informação (OMDI) influenciam as capacidades de resposta (OMR), corroborando as hipóteses H3a, H3b e H3c. Estas constatações corroboram em parte os efeitos causais entre as dimensões da Orientação para o mercado expostas por Jaworski e Kohli (1993).

A Tabela 7 sintetiza os resultados da avaliação do modelo, confirmando que a orientação para o mercado e a orientação interna estão conectadas.

Tabela 7

\section{Síntese dos Resultados das Hipóteses de Investigação}

HIPÓTESE

H1a - As capacidades de geração de informação informal do mercado interno (MIGI) têm um impacto positivo nas capacidades de divulgação interna (MIDI).

H1b - As capacidades de geração de informação formal face-to-face do mercado interno (MIFF) aumentam as capacidades de divulgação interna (MIDI).

H1c - As capacidades de geração de informação formal escrita do mercado interno (MIGF) aumentam as capacidades de divulgação interna (MIDI).

H1d - As capacidades de geração de informação informal do mercado Interno (MIGI) aumentam as capacidades de implementação de respostas (MIR).

H1e - As capacidades de geração de informação formal face-to-face do mercado interno (MIFF) aumentam as capacidades de implementação de respostas (MIR).

H1f - As capacidades de geração de informação formal escrita do mercado interno (MIGF) aumentam as capacidades de implementação de respostas (MIR).

H1g - As capacidades de divulgação interna da informação (MIDI) aumentam as capacidades de implementação de respostas (MIR).

H2a - As capacidades de geração de informação informal do mercado interno (MIGI) aumentam as capacidades de orientação para o mercado (OMPI, OMDI e OMR).

\section{RESULTADO}

Suportada

Suportada

Suportada

Não suportada

Suportada

Suportada

Suportada

Não suportada 
Tabela 7 (continuação)

\begin{tabular}{lc}
\hline HIPÓTESE & RESULTADO \\
\hline $\begin{array}{l}\text { H2b - As capacidades de geração de informação formal face-to-face do mercado } \\
\text { interno (MIFF) aumentam as capacidades de orientação para o mercado } \\
\text { (OMPI, OMDI e OMR). }\end{array}$ & Não suportada \\
$\begin{array}{l}\text { H2c - As capacidades de geração de informação formal escrita do mercado interno } \\
\text { (MIGF) aumentam as capacidades de orientação para o mercado (OMPI, }\end{array}$ & Não suportada \\
$\quad \begin{array}{l}\text { OMDI e OMR). } \\
\text { H2d - As capacidades de divulgação interna da informação (MIDI) aumentam as } \\
\text { capacidades de orientação para o mercado (OMPI, OMDI e OMR). }\end{array}$ & Suportada parcialmente \\
$\begin{array}{l}\text { H2e - As capacidades de implementação de respostas (MIR) aumentam as } \\
\text { capacidades de orientação para o mercado (OMPI, OMDI e OMR). }\end{array}$ & Suportada \\
$\begin{array}{l}\text { H3a - As capacidades de produção de informação (OMPI) aumentam as capacidades } \\
\text { de divulgação da informação (OMDI). }\end{array}$ & Suportada \\
$\begin{array}{l}\text { H3b - As capacidades de divulgação da informação (OMDI) aumentam as } \\
\text { capacidades de resposta (OMR). }\end{array}$ & Suportada \\
$\begin{array}{l}\text { H3c - As capacidades de produção de informação (OMPI) aumentam as capacidades } \\
\text { de resposta (OMR). }\end{array}$ & Suportada \\
\hline
\end{tabular}

Nota. Fonte: elaborada pelos autores.

\section{Discussão e Conclusão}

A orientação para o mercado interno favorece a atitude dos trabalhadores e tem consequências positivas na adesão dos colaboradores a práticas orientadas para o mercado, levando ao sucesso da organização no mercado externo (Ahmed \& Rafiq, 2011; Tortosa et al., 2009).

Este estudo permitiu analisar as práticas de marketing interno das empresas brasileiras, nomeadamente a geração, divulgação e implementação de respostas à informação e o seu impacto na orientação para o mercado. Foi apresentado um modelo conceitual dos relacionamentos entre os conceitos de orientação para o mercado interno e de orientação para o mercado que foi testado empiricamente.

No que diz respeito aos resultados sobre a orientação para o mercado interno, constatou-se que as capacidades de geração de informação informal do mercado interno e de informação formal face-to-face e escrita têm um impacto positivo nas capacidades de divulgação interna e nas capacidades de implementação de respostas. As três dimensões da geração de informação afetam positivamente a divulgação de informação (H1a, H1b e H1c), isto é, a disseminação da informação pelas equipes de trabalho em reuniões e contatos com os colaboradores de todos os níveis. Verificou-se igualmente que a geração de informação formal (face a face e escrita) influencia a resposta ao mercado interno (H1e e H1f), podendo levar, por exemplo, a ações corretivas e modificações das condições de trabalho. No entanto, a geração de informação informal não tem o mesmo impacto direto na implementação de respostas (H1d), sugerindo que, embora importante, a geração de informação informal é uma prática insuficiente para a empresa. É necessário que a preocupação em ouvir informalmente os colaboradores seja formalizada com práticas adequadas e que essa informação seja disseminada. Finalmente, foi estatisticamente suportada a influência da divulgação da informação no mercado interno na implementação de respostas (H1g). Com base nestas constatações, é essencial destacar a importância das interações formais e informais entre os gestores e demais trabalhadores com vista à coleta de informações sobre os desejos e necessidades desses mesmos trabalhadores. Estes resultados são semelhantes aos estudos de Jaworski e Kohli (1993) e de Lings e Greenley (2010). 
Relativamente ao impacto da orientação do mercado interno e suas dimensões na orientação para o mercado externo e respectivas dimensões, confirmou-se a importância de responder adequadamente à informação do mercado interno (H2e). É dessa forma que a orientação para o mercado interno pode ter um impacto positivo na adoção de uma cultura empresarial orientada para os clientes e no desempenho da organização. De fato, as capacidades de geração de informação informal do mercado interno não têm um impacto direto na orientação para o mercado (H2a, H2b e H2c). Quanto às capacidades de divulgação da informação, estas têm um impacto apenas numa das vertentes da orientação para o mercado externo, especificamente nas capacidades de resposta no mercado externo (H2d). Este resultado sugere que a informação gerada e divulgada internamente só terá reflexos na adoção de uma efetiva orientação para os clientes se for traduzida em ações corretivas que garantam, por exemplo, a modificação de condições de trabalho e satisfação dos funcionários. Estes resultados corroboram parcialmente os referidos nos estudos de Caruana e Calleya (1998), Gounaris et al. (2010), Lings e Greenley (2009, 2010), Naude et al. (2003), Piercy (1995) e Zaman et al. (2012).

Finalmente, no que concerne à orientação para o mercado, os resultados do presente estudo permitiram igualmente corroborar todas as hipóteses. As capacidades de produção de informação, através da pesquisa de mercado e identificação das necessidades futuras dos clientes, estão associadas às capacidades de divulgação da informação, com a partilha dessa informação pelos diferentes departamentos da empresa (H3a). Quer as capacidades de produção de informação, quer a divulgação de informação estão associadas às capacidades de resposta, através de ações de planejamento, revisão da atuação da empresa, modificações de produtos e reação a ações competitivas. Estes resultados são similares aos relatados no estudo de Jaworski e Kohli (1993).

Em termos gerais, constatou-se que a orientação para o mercado e a orientação para o mercado interno são conceitos inter-relacionados. Isso sugere que as empresas que desejam implementar com sucesso uma orientação de mercado devem adotar um foco interno complementar sobre os seus trabalhadores, garantindo que os desejos e necessidades destes também sejam satisfeitos. A implementação deste tipo de respostas terá um impacto sobre a orientação para o cliente. Para tal, o marketing interno deve garantir mecanismos para a regular sondagem, disseminação pela organização e capacidade de resposta à informação relevante sobre as expetativas e problemas

Este estudo apresenta algumas limitações. Em primeiro lugar, assinalamos as limitações decorrentes da utilização do questionário como metodologia de coleta de dados. Embora seja a metodologia maioritariamente utilizada pelos estudos nesta área, esta abordagem apresenta limitações em termos de inferência causal, dado que não permite excluir problemas como endogeneidade, omissão de variáveis importantes ou viés do método comum, pois tanto as variáveis independentes quanto as variáveis dependentes são medidas a partir dos mesmos sujeitos.

De forma a mitigar o problema do viés do método comum, foram adotadas medidas ex-ante e medidas ex-post, de acordo com a sugestão de Chang, Witteloostuijn e Eden (2010). Assim, os respondentes foram assegurados do anonimato e confidencialidade das suas respostas e da importância de expressarem a sua opinião verdadeira. Procedeu-se também ao teste de um fator de Harman, que indicou a presença de 9 fatores com autovalor superior a 1, sendo que nenhum dos quais capturou uma parte significativa da variância total.

É importante referir também as limitações decorrentes da técnica de amostragem utilizada, o que requer alguma prudência na generalização dos resultados. Porém, apesar de não ser representativa, a dimensão da nossa amostra é adequada para avaliar o número de variáveis do modelo (Hair, Black, Babin, Anderson, \& Tatham, 2009). Assim, representa um contributo assinalável para compreender as práticas de marketing interno das empresas brasileiras, sobretudo devido à dificuldade de obtenção de dados em contextos organizacionais.

Esforços futuros devem continuar a avançar nossa compreensão do conceito de marketing interno, os meios para a sua mensuração e as suas implicações internas e externas para as organizações. Compreender o papel das práticas de marketing interno no desenvolvimento de um ambiente de trabalho propício a uma verdadeira orientação para o mercado, garantindo que os colaboradores tenham a 
motivação e incentivos para transformar as suas interações com os clientes em oportunidades de aprendizagem e de melhoria para a organização, permanece um desígnio para a investigação nesta área. Estudos futuros poderão incluir pesquisas com uma amostra representativa, abordagens qualitativas com uma maior diversidade de fontes de informação e a utilização de estudos longitudinais permitindo uma análise dinâmica das relações entre os construtos.

\section{Referências}

Ahmed, P. K., \& Rafik, M. (2011). Internal marketing: the tools and concepts for customer-focused management. London: Routledge.

Atuahene-Gima, K. (1996). Market orientation and innovation. Journal of Business Research, 35(2), 93-103. doi: 10.1016/0148-2963(95)00051-8

Berry, L., Hensel, J., \& Burke, M. (1976). Improving retailer capability for effective consumerism response. Journal of Retailing, 52(3), 3-14.

Caruana, A., \& Calleya, P. (1998). The effect of internal marketing on organisational commitment among retail bank managers. International Journal of Bank Marketing, 16(3), 108-116. doi: $10.1108 / 02652329810213510$

Cassundé, F. R. S. A., Cassundé, N. F., Jr., Farias, S. A., \& Mendonça, J. R. C. de (2014). What is being said about internal marketing? A study of the Brazilian business administration academic production. Brazilian Business Review, 11(5), 49-71. doi: 10.15728/bbr.2014.11.5.3

Chang, S.-J., Witteloostuijn, A. van, \& Eden, L. (2010). From the editors: common method variance in international business research. Journal of International Business Studies, 41(2), 178-184. doi: $10.1057 /$ jibs.2009.88

Deshpandé, R., Farley, J. U., \& Webster, F. R., Jr. (1993). Corporate culture, customer orientation, and innovativeness in Japanese firms: a quadrad analysis. Journal of Marketing, 57(1), 23-27. doi: $10.2307 / 1252055$

Farias, S. A. (2010). Internal marketing (IM): a literature review and research propositions for service excellence. Brazilian Business Review, 7(2), 99-115. doi: 10.15728/bbr.2010.7.2.6

George, W. R. (1990). Internal marketing and organizational behavior: a partnership in developing customer-conscious employees at every level. Journal of Business Research, 20(1), 63-70. doi: 10.1016/0148-2963(90)90043-D

Gounaris, S. (2006). Internal-market orientation and its measurement. Journal of Business Research, 59(4), 432-448. doi: 10.1016/j.jbusres.2005.10.003

Gounaris, S. (2008). Antecedents of internal marketing practice: some preliminary empirical evidence. International Journal of Service Industry Management, 19(3), 400-434. doi: $10.1108 / 09564230810875039$

Gounaris, S., Vassilikopoulou, A., \& Chatzipanagiotou, K. C. (2010). Internal-market orientation: a misconceived aspect of marketing theory. European Journal of Marketing, 44(11/12), 1667-1699. doi: 10.1108/03090561011079837

Grönroos, C. (1994). From marketing mix to relationship marketing: towards a paradigm shift in marketing. Management Decision, 32(2), 4-20. doi: 10.1108/00251749410054774 
Grönroos, C. (1995). Relationship marketing: the strategy continuum. Journal of the Academy of Marketing Science, 23(4), 252-255. doi: 10.1007/BF02893863

Hair, J. F., Black, W. C., Babin, B. J., Anderson, R. E., \& Tatham, R. L. (2009). Multivariate data analysis. Upper Saddle River: Prentice Hall.

Harzing, A.-W. (2000). Cross-national industrial mail surveys why do response rates differ between countries? Industrial Marketing Management, 29(3), 243-254. doi: 10.1016/S00198501(99)00063-2

Hemais, M. W., Oliveira, L. B. de, \& Casotti, L. M. (2013). Marketing interno e gestão de pessoas: estudos de caso em empresas de serviços. Revista Gestão \& Tecnologia, 13(3), 101-127.

Jaworski, B., \& Kohli, A. (1993). Market orientation: antecedents and consequences. Journal of Marketing, 57(3), 53-70. doi: 10.2307/1251854

Kohli, A. K., \& Jaworski, B. J. (1990). Market orientation: the construct, research propositions, and managerial implications. Journal of Marketing, 54(2), 1-18. doi: 10.2307/1251866

Kohli, A. K., Jaworski, B. J., \& Kumar, A. (1993). MARKOR: a measure of market orientation. Journal of Marketing Research, 30(4), 467-477. doi: 10.2307/3172691

Liao, S.-H., Chang, W.-J., Wu, C.-C., \& Katrichis, J. M. (2011). A survey of market orientation research (1995-2008). Industrial Marketing Management, 40(2), 301-310. doi: 10.1016/j.indmarman.2010.09.003

Lings, I. N. (2004). Internal market orientation: construct and consequences. Journal of Business Research, 57(4), 405-413. doi: 10.1016/S0148-2963(02)00274-6

Lings, I. N., \& Greenley, G. E. (2005). Measuring internal market orientation. Journal of Service Research, 7(3), 290-305. doi: 10.1177/1094670504271154

Lings, I. N., \& Greenley, G. E. (2009). The impact of internal and external market orientations on firm performance. Journal of Strategic Marketing, 17(1), 41-53. doi: 10.1080/09652540802619251

Lings, I. N., \& Greenley, G. E. (2010). Internal market orientation and market-oriented behaviours. Journal of Service Management, 21(3), 321-343. doi: 10.1108/09564231011050788

Narver, J., \& Slater, S. (1990). The effect of a market orientation on business profitability. Journal of Marketing, 54(4), 20-35. doi: 10.2307/1251757

Naude, P., Desai, J., \& Murphy, J. (2003). Identifying the determinants of internal marketing orientation. European Journal of Marketing, 37(9), 1205-1220. doi: 10.1108/0390560310486951

Paim, N. A., Lara, J. E., \& Christino, J. M. M. (2011). Relações quantitativas entre orientação para o mercado, desempenho organizacional e a percepção externa de sucesso em instituições de pesquisas tecnológicas afiliadas à ABIPTI. Revista de Administração Contemporânea, 15(6), 1059-1077. Recuperado de http://www.scielo.br/pdf/rac/v15n6/06.pdf. doi: 10.1590/S141565552011000600006

Piercy, N. (1995). Customer satisfaction and the internal market: marketing our customers to our employees. Journal of Marketing Practice, 1(1), 22-44. doi: 10.1108/EUM0000000003878

Raaij, E., \& Stoelhorst, J. (2008). The implementation of a market orientation: a review and integration of the contributions to date. European Journal of Marketing, 42(11/12), 1265-1293. doi: $10.1108 / 03090560810903673$ 
Rafiq, M., \& Ahmed, P. Q. (1993). The scope of internal marketing: defining the boundary between marketing and human resource management. Journal of Marketing Management, 9(3), 219-232. doi: 10.1080/0267257X.1993.9964234

Rafiq, M., \& Ahmed, P. Q. (2000). Advances in the internal marketing concept: definition, synthesis and extension. Journal of Services Marketing, 14(6), 449-462. doi: 10.1108/08876040010347589

Ruekert, R. W. (1992). Developing a market orientation: an organizational strategy perspective. International Journal of Research in Marketing, 9(3), 225-245. doi: 10.1016/01678116(92)90019-H

Sampaio, C. (2000). Relação entre orientação para o mercado e performance empresarial em empresas de varejo de vestuário do Brasil (Tese de doutorado). Universidade Federal do Rio Grande do Sul, Porto Alegre, RS, Brasil.

Shapiro, B. P. (1988). What the hell is 'market oriented'? Harvard Business Review, 66(6), 119-125.

Tortosa, V., Moliner, M. A., \& Sánchez, J. (2009). Internal market orientation and its influence on organisational performance. European Journal of Marketing, 43(11/12), 1435-1456. doi: $10.1108 / 03090560910989975$

Zaman, K., Javaid, N., Arshad, A., \& Bibi, S. (2012). Impact of internal marketing on market orientation and business performance. International Journal of Business and Social Science, 3(12), 76-88.

\section{Dados dos Autores}

Iara Dantas Cordeiro de Morais

Universidade do Minho, Escola de Economia e Gestão, Campus de Gualtar, 4710-057, Braga, Portugal. E-mail: iara.dcordeiro@gmail.com

Ana Maria Soares

Universidade do Minho, Escola de Economia e Gestão, Campus de Gualtar, 4710-057, Braga, Portugal. E-mail: amsoares@eeg.uminho.pt 